\title{
1 Genetic selection for small molecule production in competitive microfluidic
}

\section{2 droplets}

$4 \quad$ Larry J. Millet ${ }^{1,2}$, Jessica M. Velez ${ }^{1}$, Joshua K. Michener ${ }^{1, *}$

5 (1) Biosciences Division, Oak Ridge National Laboratory, Oak Ridge, TN, 37830; (2) The Joint

6 Research Activity Unit of The Bredesen Center for Interdisciplinary Research and Graduate

7 Education, University of Tennessee, Knoxville, TN, 37996.

$9 \quad(*)$ Correspondence should be addressed to JKM (michenerjk@ornl.gov)

11 This manuscript has been authored by UT-Battelle, LLC under Contract No. DE-AC05-

12 00OR22725 with the U.S. Department of Energy. The United States Government retains and the

13 publisher, by accepting the article for publication, acknowledges that the United States

14 Government retains a non-exclusive, paid-up, irrevocable, world-wide license to publish or

15 reproduce the published form of this manuscript, or allow others to do so, for United States

16 Government purposes. The Department of Energy will provide public access to these results of

17 federally sponsored research in accordance with the DOE Public Access Plan

18 (http://energy.gov/downloads/doe-public-access-plan). 
20 Abstract

21 Biosensors can be used to screen or select for small molecule production in engineered microbes.

22 However, mutations to the biosensor that interfere with accurate signal transduction are common,

23 producing an excess of false positives. Strategies have been developed to avoid this limitation by

24 physically separating the production pathway and biosensor, but these approaches have only

25 been applied to screens, not selections. We have developed a novel biosensor-mediated selection

26 strategy using competition between co-cultured bacteria. When applied to biosynthesis of cis, cis-

27 muconate, we show that this strategy yields a selective advantage to producer strains that

28 outweighs the costs of production. By encapsulating the competitive co-cultures into

29 microfluidic droplets, we successfully enriched for muconate-producing strains from a large

30 population of control non-producers. Facile selections for small molecule production will

31 increase testing throughput for engineered microbes and allow for the rapid optimization of

32 novel metabolic pathways. 


\section{Introduction}

Engineered microbes can produce a range of valuable bioproducts, providing the basis for a

35

renewable bioeconomy ${ }^{1}$. However, initial strain construction efforts are often inefficient, and the cost and effort required to optimize a proof-of-concept pathway can preclude further development. New strategies for constructing libraries of mutant strains have accelerated the design and build phases of the engineering $\operatorname{cycle}^{2-4}$, but these efforts are limited by the throughput of methods for identifying improved variants. Demonstrations of new library construction methods frequently use phenotypes that can be linked to microbial growth or to production of an easily-identifiable molecule, such as visibly-pigmented compounds. Using similar approaches to engineer strains that produce higher concentrations of an arbitrary small molecule is challenging.

One option for identifying rare variants from a mutant library is to include a biosensor, such as a metabolite-responsive transcription factor or regulatory RNA, that reports on production of the target small molecule ${ }^{5,6}$. These biosensors can be highly effective, but they also come with several limitations. Most notably, the biosensor is typically expressed from the same cell that is being engineered or evolved. As a result, mutations can occur in the sensor that disrupt the desired regulation, producing false positive signals that require significant additional screening ${ }^{6}$. An intracellular biosensor also reports on the intracellular small molecule concentration. A product that is rapidly exported may not show a differential signal as the production rate changes. Similarly, a product that is rapidly imported may produce crosstalk between neighboring cells. Finally, biosensors can be linked to selectable markers such as antibiotic resistance genes or screenable markers such as fluorescent proteins. Selections typically enable larger library sizes and require less infrastructure, but also amplify any concerns about false 
positives during the selection.

Several strategies have been proposed to overcome these challenges using biosensors to screen mutant libraries. Avoiding false positives requires separating the biosensor from the strain being engineered in order to limit mutation accumulation in the biosensor. After the two functions are separated, though, measurements of productivity then require either disruptive methods to reintroduce the sensor into the producer strains ${ }^{7}$ or some form of spatial structure to prevent crosstalk between members of the library. Initial efforts often used colonies spread on petri dishes, which limited the assay throughput ${ }^{8}$. More recent approaches rely on encapsulation of cells inside microfluidic droplets ${ }^{9-12}$. By physically separating individual cells into distinct droplets, interactions between cells can be eliminated. However, all of these approaches depend on quantitative screens, limiting throughput and requiring fluorescence-activated cell sorters or on-chip droplet sorters to isolate desired droplets. A biosensor-driven selection strategy would simplify the process of isolating improved variants from a mutant library while expanding the potential library size.

We have developed a selection strategy that uses competition between bacterial strains within microfluidic droplets to select for small molecule biosynthesis. Production of the target small molecule inhibits growth of a sensitive competitor and provides a selective advantage to the producer (Figure 1A). We show that competitor strains can be rationally engineered to be inhibited by a target small molecule, that production of the target provides an advantage to the producer, and that spatial structure is required to prevent interactions between producer cells. By combining these elements, we enriched true producer strains from a background of fastergrowing non-producers. These high-through selections for small molecule production will enable rapid optimization of engineered microbes. 


\section{Results and Discussion}

\section{Strain design and construction}

To establish a microbial competition mediated by small molecule production, two different

A Producer
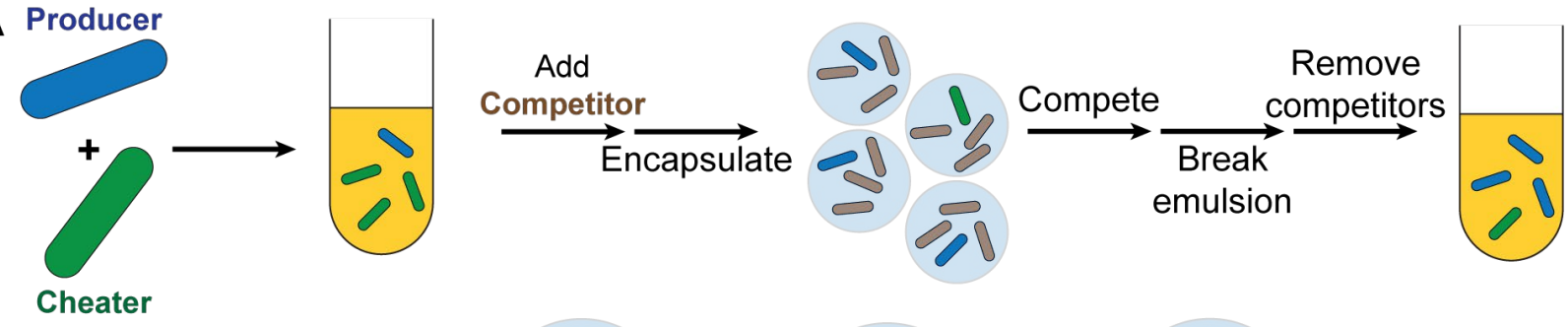

B

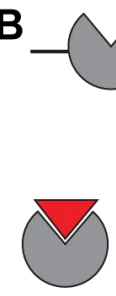

c

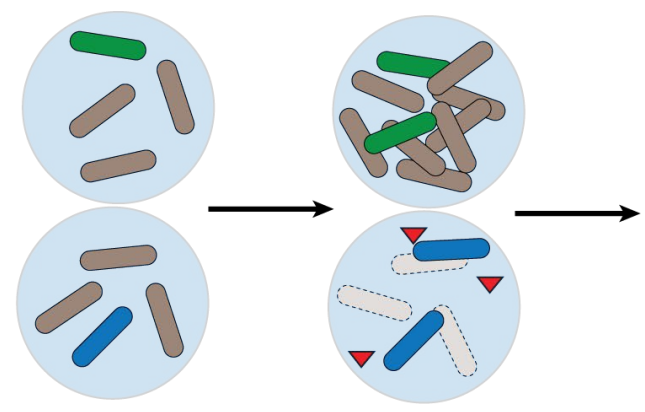

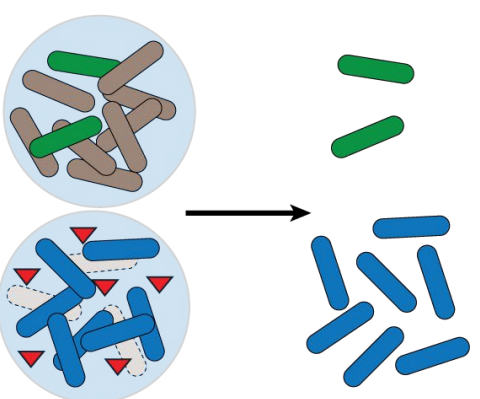

Figure 1: Competitive microfluidic droplets enable selections for small molecule production.
(A) A simplified population contains a mix of producer strains (blue) that synthesize the desired

small molecule and cheater strains (green) that do not. This population is then combined with the competitor strain (brown) and encapsulated into microfluidic droplets where the competitor is inhibited by the target molecule. The mixed cultures are grown to saturation, the emulsion is broken, and the producer cells increase in frequency due to the fitness benefit of eliminating the competitor strain. (B) The competitor strain is inhibited by the target molecule (red triangle) due 
to regulation of the counterselectable marker $r p s L$ by the biosensor. In the presence of the target molecule, production of RpsL sensitizes the competitor strain to streptomycin. (C) In a droplet containing a cheater, the competitor strain outcompetes the cheater and limits the yield.

Conversely, in a droplet containing a producer, production of the target molecule blocks competitor growth and increases the yield of the producer strain.

The selection system consists of two strains, one engineered to produce muconate and the other engineered to be inhibited by muconate. To build a suitable muconate-producing strain, we started with $P$. putida CJ102, which was been previously engineered to synthesize muconate from lignin-derived aromatic compounds ${ }^{13}$. The strain was further modified by deletion of the adhesin $\operatorname{lap} A$, to prevent biofilm formation that otherwise interfered with independent segregation $^{14}$; deletion of a component of the type 6 secretion system $t s S A 1$, to eliminate an alternative killing mechanism ${ }^{15}$; deletion of the glucose dehydrogenase $g c d$, to prevent accumulation of a potential cross-feeding metabolite ${ }^{16,17}$; and introduction of a streptomycinresistance marker ${ }^{18}$. The resulting strain, JMP15, was then further modified by deletion of the phenylpropanoid-CoA synthetase $f c s^{19}$ to produce a control strain, JMP26, that is incapable of converting phenylpropanoids into muconate (Supplementary Figure 1A).

For the muconate-inhibited strain, we introduced a counterselection module into a streptomycin-resistant strain of $E$. coli. The counterselection module consists of a constitutive promoter driving expression of an operon containing a muconate transporter and a muconateresponsive transcription factor, combined with a regulated promoter driving expression of a counterselectable marker (Supplementary Figure 1B). We selected the mucK muconate 
118 from Acinetobacter baylyi ${ }^{20}$, and then expressed a streptomycin-sensitive allele of $r p s L$ from the 119 BenM promoter. The counterselection module was synthesized de novo and cloned into pET-9a.

When the resulting plasmid was transformed into streptomycin-resistant E. coli REL606,

121 addition of muconate inhibited growth in a dose-dependent fashion, with growth completely blocked at $10 \mathrm{mg} / \mathrm{L}$ muconate (Figure 2).

123

124

125

126

127

128

129

130

131

132

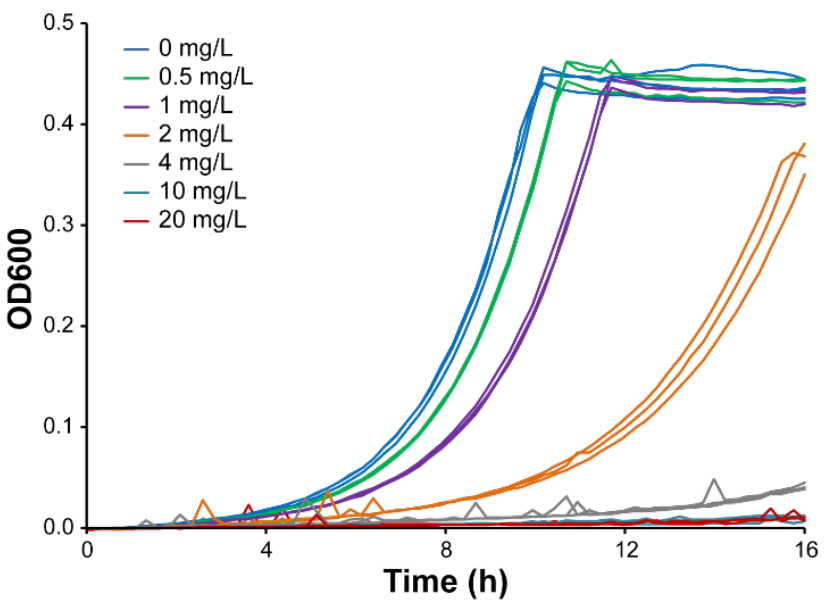

Figure 2: A muconate-responsive counterselection module demonstrates dose-dependent growth inhibition. Streptomycin-resistant REL606 containing plasmid pJM242 was grown in minimal medium containing ampicillin, streptomycin, and the indicated concentration of muconate. Three biological replicates are shown.

\section{Muconate production is costly but provides a competitive advantage against sensitive $E$.}

coli

The engineered producer strain, JMP15, can convert coumarate to muconate in a series of reactions that conserve little usable energy. Consequently, the strain grows more slowly in minimal media containing glucose when coumarate is also added (Figure 3A). The control strain, JMP26, does not show a growth difference.

We then grew the producer and control strains of $P$. putida in co-culture with the muconate- 

replicates.

sensitive $E$. coli. After growth, the $P$. putida concentration was measured by plating on selective agar. When exogenous muconate was added, both $P$. putida strains showed large increases in yield (Figure 3B). After addition of coumarate, the muconate-producing strain JMP15 showed a small but significant fold increase in yield of $1.9 \pm 0.2$ while the yield of the control strain was unchanged. When grown in competition with a muconate-sensitive E. coli, muconate production is costly to the cell, but the benefits of inhibiting $E$. coli growth offset the costs.

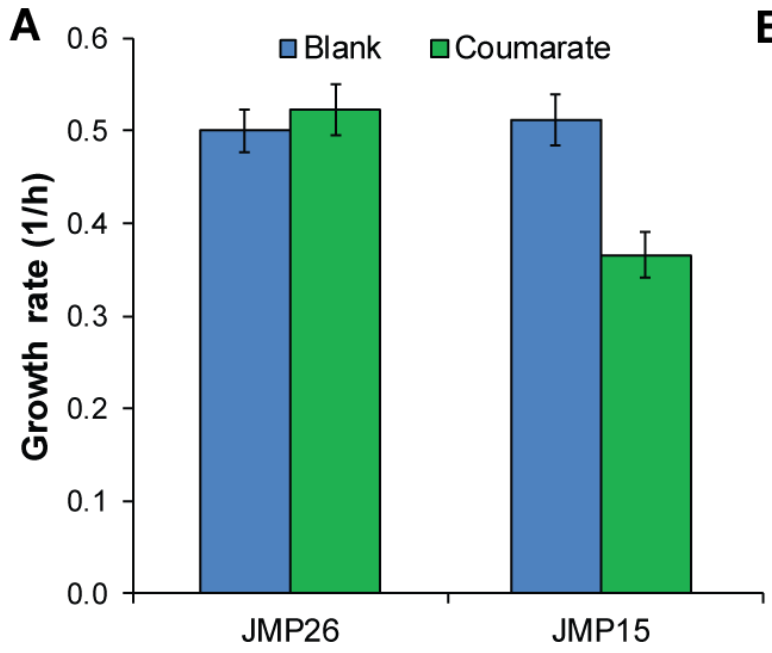

$(\Delta f c s)$

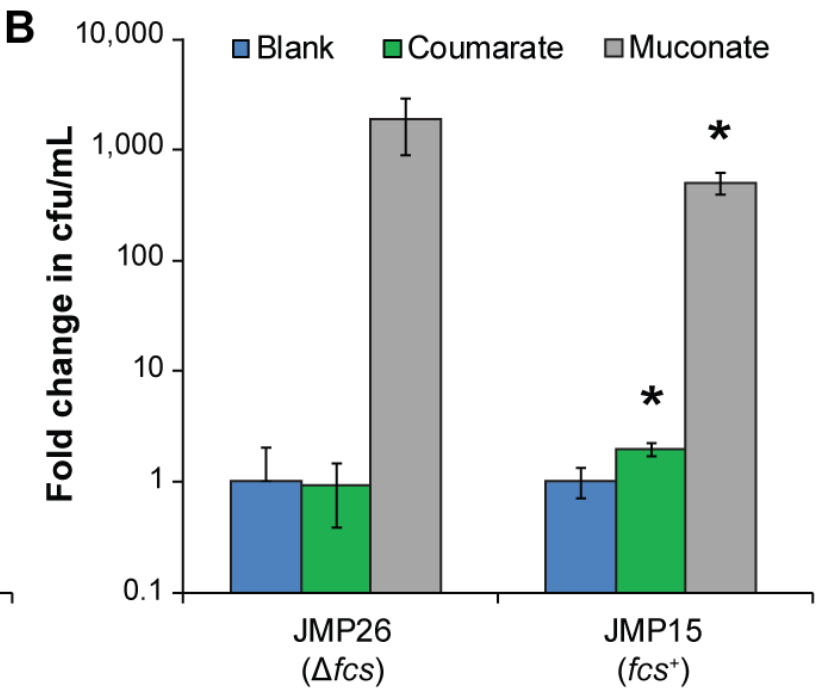

Figure 3: The benefits of muconate production outweigh the costs. (A) Addition of coumarate, which can be converted into muconate, decreases the growth rate of JMP15. Deletion of the gene encoding the first enzyme in coumarate metabolism, the CoA synthetase $f c s$, eliminates this effect. (B) When grown in competition with E. coli that are sensitive to muconate, the addition of coumarate provides a selective advantage only for the strain able to convert it into muconate. ${ }^{*}: p$ $<0.05$, two-tailed t-test. Error bars show one standard deviation, calculated from three biological 
153

154

155

156

157

158

159

160

161

A

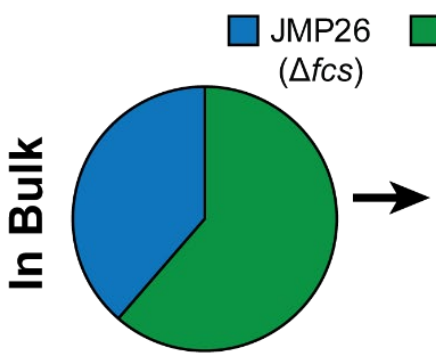

Inoculum

B

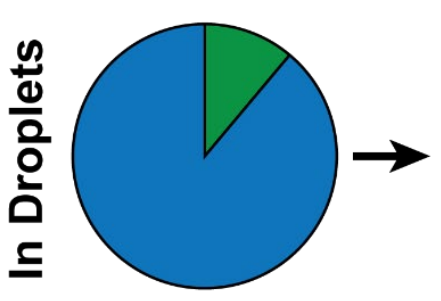

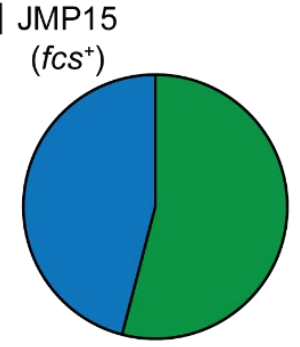

Post-growth

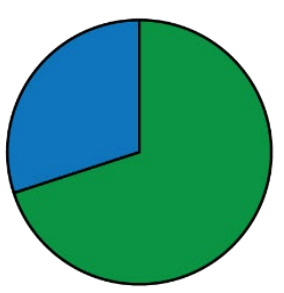

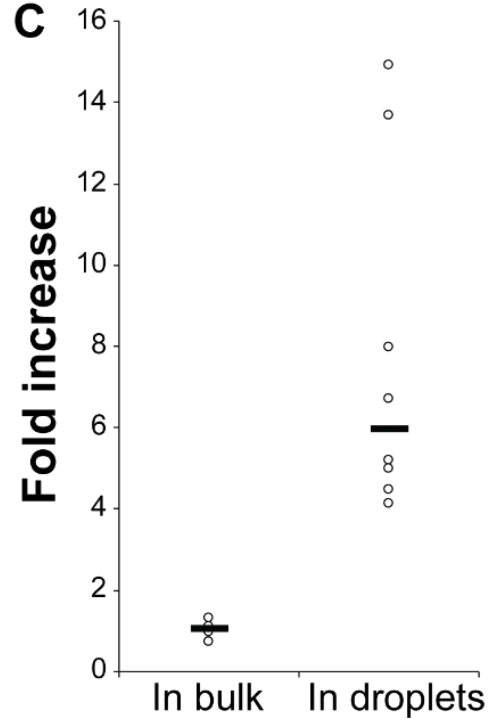

168 Figure 4: Spatial structure enables muconate selection. (A) A mixture of control and producer 
strains were competed against muconate-sensitive $E$. coli in test tubes. The fraction of JMP15 in the population did not change significantly. (B) When the mixed cultures were encapsulated into microfluidic droplets, a single night of growth enriched for muconate producers. One representative population ratio is shown for each condition. (C) The fold increase in JMP15 relative to JMP26 was calculated for each competition culture. Each point represents a single biological replicate, and each set of competitions was repeated on at least two days. White circles: individual cultures. Black bars: Distribution median.

This selection strategy has several key advantages. By separating the counterselection module into a separate cell, mutations that prevent proper biosensor function yield false negatives rather than false positives, by preventing producers from gaining the expected benefits of muconate production. When selecting for rare phenotypes, false negatives are much more easily tolerated than false positives. Similarly, the competitor strain can be eliminated and reintroduced after every round of growth, preventing the accumulation of strains with mutated biosensors.

This system also streamlines the process of using biosensors in diverse bacteria. The producer and competitor strains can be entirely different species, individually chosen for specific purposes. Rather than engineering the muconate biosensor into $P$. putida, we were able to leave it in the more-tractable $E$. coli host. This muconate-sensitive competitor can then be used with any muconate producing strain that has compatible culture conditions. The producer strain, meanwhile, has no remnant of the biosensor that needs to be removed later. We also note that the biosensor is not strictly necessary, as long as a competitor strain can be identified or engineered that is inhibited by the product of interest. Finally, the validation of a new biosensor is 
192 simplified. Frequently, biosensors are validated through exogenous addition of small molecules

193 and then used to sense compounds produced intracellularly. Here, the competitor strain is

194 intended to respond to exogenous products, so testing and application are performed under

195 equivalent conditions.

The selective pressures that result from this approach are complex. An increase in either

197 growth rate or muconate production would be expected to provide a selective advantage. In 198 general, we expect this complex selective pressure to be a benefit of this approach, since the

199 parallel selection for growth rate prevents the accumulation of mutations with strong fitness costs

200 and will yield robust strains. The selective pressure can also be tuned in multiple ways, including

201 modifying the sensitivity of the biosensor to its target, changing the initial population ratio of

202 producers and competitors, or engineering the competitor to grow more or less quickly.

While the initial selective advantage for a single round of growth in droplets was modest,

$5.8 \pm 1.5$-fold, this represents a clear selection against nonproductive mutations. Mutant strains larger fold enrichment.

New methods for strain and library construction have expanded the opportunities for largescale biological design. However, testing the resulting strains currently limits throughput. To

209 address this bottleneck, we have developed a flexible biosensor-driven selection strategy using bacterial competition in microfluidic droplets. Using this approach, libraries generated through a

211 variety of methods can be rapidly tested to identify rare variants that improve productivity. By 


\section{Materials and Methods}

\section{Media and chemicals}

217 All chemicals were from Sigma-Aldrich unless otherwise noted. Strains were routinely

218 propagated in LB or M9 minimal medium containing $2 \mathrm{~g} / \mathrm{L}$ glucose. Antibiotics were $50 \mu \mathrm{g} / \mathrm{mL}$

219 kanamycin, $100 \mu \mathrm{g} / \mathrm{mL}$ ampicillin, and $50 \mu \mathrm{g} / \mathrm{mL}$ streptomycin. Stock solutions of muconate and

220 coumarate at $100 \mathrm{mg} / \mathrm{mL}$ were made fresh in DMSO and diluted to the appropriate

221 concentrations as needed. Microfluidic droplets used HFE-7500 (3M, St. Paul, MN) as the bulk

222 phase and 008-FluoroSurfactant (RAN Biotechnologies, Beverly, MA) as the surfactant.

\section{Genetic manipulation}

Strains JMP15 and JMP26 contain multiple mutations relative to the parental strain CJ102.

226 For gene deletions, the appropriate deletion construct, containing approximately $500 \mathrm{bp}$ of

227 homology on both sides of the gene to be deleted, were synthesized de novo (IDT, Skokie, IL)

228 and assembled into $\mathrm{pK} 18 \mathrm{mobsacB}^{21}$ using the HiFi Assembly Master Mix (NEB, Ipswich, MA).

229 The resulting plasmids were transformed into $P$. putida by electroporation, followed by selection

230 on LB + kanamycin and counterselection on YT $+25 \%$ sucrose $^{22}$. Gene deletions were verified

231 by colony PCR and Sanger sequencing (Eurofins Genomics, Louisville, KY). To introduce the

232 streptomycin resistance marker, plasmids pUX-BF13 and pBK-miniTn7-gfp3 were

233 cotransformed into the recipient $P$. putida strain by electroporation, followed by selection on LB

$234+$ kanamycin.

\section{Growth curves}

237 Strains for growth curve experiments were grown to saturation overnight at $30{ }^{\circ} \mathrm{C}$ in $\mathrm{M} 9+2$ 
$\mathrm{g} / \mathrm{L}$ glucose + ampicillin. The cultures were then diluted 100-fold into fresh medium and grown in 48 -well plates in an Epoch 2 plate reader (BioTek, Winooski, VT) at $30{ }^{\circ} \mathrm{C}$. Growth rates were calculated using CurveFitter software ${ }^{23}$.

\section{Bulk competition assays}

Strains JMP15, JMP26, and REL606+pJM242 were grown to saturation overnight at $30^{\circ} \mathrm{C}$ in $\mathrm{M} 9+2 \mathrm{~g} / \mathrm{L}$ glucose + ampicillin. The cultures were washed once and then diluted into $5 \mathrm{~mL}$ of fresh M9 $+4 \mathrm{~g} / \mathrm{L}$ glucose + ampicillin + streptomycin containing the relevant substrate as indicated. Inoculation densities were OD 0.01 for JMP15 or JMP26, and 0.3 for REL606+pJM242. For mixed P. putida cultures, the inoculation densities for JMP15 and JMP26 were 0.005 , and the inoculum cultures were plated on LB + kanamycin as described below to determine the initial population ratio. Cultures were grown overnight at $30^{\circ} \mathrm{C}$, then diluted and plated on LB + kanamycin. For pure culture experiments, colonies were counted and adjusted for the dilution factor. For mixed culture experiments, individual colonies were isolated and genotyped by colony PCR of the $f c s$ locus.

\section{Droplet competition assays}

Droplet competition assays were performed in a similar fashion as bulk assays. The $P$. putida inoculation densities were decreased to 0.002 for pure cultures or 0.001 for each mixed culture, to produce an average of one $P$. putida per 10 droplets. The $E$. coli inoculation density was maintained at 0.15 , yielding an average of approximately $6 E$. coli per droplet. Initial population ratios were determined by plating dilutions on $\mathrm{LB}+$ kanamycin agar.

Droplet encapsulation was performed on a Dolomite $\mu$ Encapsulator system using a $50 \mu \mathrm{m}$ 
261 fluorophilic droplet chip, according to the manufacturer's directions. The P. putida and E. coli

262 cell suspensions were placed in separate channels of the reservoir immediately before

263 encapsulation. Flow rates of the driving fluids for $P$. putida and E. coli were set to $7 \mu \mathrm{L} / \mathrm{min}$ and

264 the surfactant-in-oil solution was held constant at $22 \mu \mathrm{L} / \mathrm{min}$. Stability of the droplet generation

265 process was visually monitored to validate each run to completion. A typical run generated

266 approximately three million droplets with an average diameter of $60 \mu \mathrm{m}$. After one culture

267 reservoir was exhausted, the collection vial was removed, immediately capped, inspected for

268 quality (no visually distinguishable large droplets observed) and emulsion quantity $(\sim 500 \mu \mathrm{L}$

269 total, $200 \mu \mathrm{L}$ emulsion layer), and incubated at $30{ }^{\circ} \mathrm{C}$ overnight.

After overnight growth, the emulsion was broken through the addition of $200 \mu \mathrm{L}$ of

271 perfluorooctanol. The mixture was gently mixed by flicking the tube 5 times, then allowed to

272 settle for $5 \mathrm{~min}$. The top aqueous layer $(\sim 120 \mu \mathrm{L})$ was removed into a new sterile microfuge

273 tube and serial dilutions $\left(10^{-1}\right.$ to $\left.10^{-3}\right)$ plated onto LB + kanamycin agar. Following overnight

274 growth at $30^{\circ} \mathrm{C}$, densities were determined through colony counting, and population ratios by

275 colony PCR of the $f c s$ locus as described above.

276

$277 \quad$ Tables

\begin{tabular}{|l|l|l|}
\hline Strain & Genotype & Source \\
\hline REL606 & $\mathrm{F}^{-}$tsx467(Am) araA230 lon $r p s L 227\left(\mathrm{Sm}^{\mathrm{R}}\right) h s d R$ & 24 \\
\hline CJ102 & P. putida KT2440 $\Delta$ catRBC::Ptac:catA $\Delta p c a H G:: P t a c: a r o Y$ & 25 \\
\hline JMP9 & CJ102 $\Delta l a p A$ & This work \\
\hline JMP10 & CJ102 $\Delta l a p A \Delta t s s A 1$ & This work \\
\hline JMP14 & CJ102 $\Delta l a p A \Delta t s s A 1 \Delta g c d$ & This work \\
\hline JMP15 & CJ102 $\Delta l a p A \Delta t s s A 1 \Delta g c d \mathrm{Km}^{\mathrm{R}} \mathrm{Sm}^{\mathrm{R}} \mathrm{GFP}$ & This work \\
\hline JMP25 & CJ102 $\Delta l a p A \Delta t s s A 1 \Delta g c d \Delta f c s$ & This work \\
\hline JMP26 & CJ102 $\Delta l a p A \Delta t s s A 1 \Delta g c d \Delta f c s \mathrm{Km}^{\mathrm{R}} \mathrm{Sm}^{\mathrm{R}} \mathrm{GFP}$ & This work \\
\hline
\end{tabular}

Table 1: Strains used in this work. 


\begin{tabular}{|l|l|l|}
\hline Plasmid & Genotype & Source \\
\hline pUX-BF13 & $m o b^{+} \mathrm{Tn} 7$ transposase $\mathrm{Ap}^{\mathrm{R}}$ & 18 \\
\hline pBK-miniTn7-gfp3 & $\mathrm{Km}^{\mathrm{R}} \mathrm{Cm}^{\mathrm{R}} \mathrm{Sm}^{\mathrm{R}} \mathrm{Ap}^{\mathrm{R}}$ mob $^{+} g f p$ & 18 \\
\hline pJV1 & $\mathrm{Km}^{\mathrm{R}} s a c B \mathrm{mob}^{+} \Delta t s s A 1$ & This work \\
\hline pJE364 & $\mathrm{Km}^{\mathrm{R}} s a c B m o b^{+} \Delta l a p A$ & Unpublished \\
\hline pJE365 & $\mathrm{Km}^{\mathrm{R}} s a c B m o b^{+} \Delta g c d$ & Unpublished \\
\hline pJM242 & $\mathrm{pBR}^{2} 22 \mathrm{Ap}^{\mathrm{R}}$ apFab61-mucK-benM pbenM-rpsL & This work \\
\hline pJM384 & $\mathrm{Km}^{\mathrm{R}} s a c B m o b^{+} \Delta f c s$ & This work \\
\hline
\end{tabular}

Table 2: Plasmids used in this work

\section{Acknowledgements}

\section{Funding Information}

This work was supported by the Laboratory Directed Research and Development program at the Oak Ridge National Laboratory.

\section{References}

292 1. Department of Energy, U. S. 2016 Billion-Ton Report: Advancing domestic resources for a thriving bioeconomy, Volume 1: Economic availability of feedstocks. (2016). protein, metabolic and genome engineering. Nat. Biotechnol. 35, 48-55 (2016). 
nucleotides in a tunable window. Nature 560, 248-252 (2018).

298 4. Wang, H. H. et al. Programming cells by multiplex genome engineering and accelerated evolution. Nature 460, 894-898 (2009).

$300 \quad 5 . \quad$ Tang, S.-Y. \& Cirino, P. C. Design and Application of a Mevalonate-Responsive Regulatory Protein. Angew. Chemie Int. Ed. 50, 1084-1086 (2011).

302 6. Michener, J. K. \& Smolke, C. D. High-throughput enzyme evolution in Saccharomyces cerevisiae using a synthetic RNA switch. Metab. Eng. 14, 306-316 (2012).

$3047 . \quad$ Esvelt, K. M., Carlson, J. C. \& Liu, D. R. A system for the continuous directed evolution 305 of biomolecules. Nature 472, 499-503 (2011).

8. $\quad$ Pfleger, B. F., Pitera, D. J., Newman, J. D., Martin, V. J. J. \& Keasling, J. D. Microbial sensors for small molecules: Development of a mevalonate biosensor. Metab. Eng. 9, 3038 (2007).

9. Agresti, J. J. et al. Ultrahigh-throughput screening in drop-based microfluidics for directed evolution. Proc. Natl. Acad. Sci. U. S. A. 107, 4004-9 (2010).

311 10. Wang, B. L. et al. Microfluidic high-throughput culturing of single cells for selection based on extracellular metabolite production or consumption. Nat. Biotechnol. 32, 473478 (2014).

11. Siedler, S. et al. Development of a bacterial biosensor for rapid screening of yeast $p$ coumaric acid production. ACS Synth. Biol. acssynbio.7b00009 (2017). doi:10.1021/acssynbio.7b00009

317 12. Abatemarco, J. et al. RNA-aptamers-in-droplets (RAPID) high-throughput screening for secretory phenotypes. Nat. Commun. 8, 332 (2017).

319 13. Vardon, D. R. et al. cis,cis-Muconic acid: separation and catalysis to bio-adipic acid for 
nylon-6,6 polymerization. Green Chem. 18, 3397-3413 (2016).

321

322

323

324

325

326

327

328

329

330

331

332

333

334

335

336

337

338

339

340

14. Espinosa-Urgel, M., Salido, A. \& Ramos, J. L. Genetic analysis of functions involved in adhesion of Pseudomonas putida to seeds. J. Bacteriol. 182, 2363-9 (2000).

15. Bernal, P., Allsopp, L. P., Filloux, A. \& Llamas, M. A. The Pseudomonas putida T6SS is a plant warden against phytopathogens. ISME J. 11, 972-987 (2017).

16. del Castillo, T. et al. Convergent peripheral pathways catalyze initial glucose catabolism in Pseudomonas putida: genomic and flux analysis. J. Bacteriol. 189, 5142-52 (2007).

17. Eisenberg, R. C. \& Dobrogosz, W. J. Gluconate metabolism in Escherichia coli. J. Bacteriol. 93, 941-9 (1967).

18. Koch, B., Jensen, L. E. \& Nybroe, O. A panel of Tn7-based vectors for insertion of the gfp marker gene or for delivery of cloned DNA into Gram-negative bacteria at a neutral chromosomal site. J. Microbiol. Methods 45, 187-195 (2001).

19. Nelson, K. E. et al. Complete genome sequence and comparative analysis of the metabolically versatile Pseudomonas putida KT2440. Environ. Microbiol. 4, 799-808 (2002).

20. Rogers, J. K. \& Church, G. M. Genetically encoded sensors enable real-time observation of metabolite production. Proc. Natl. Acad. Sci. U. S. A. 113, 2388-93 (2016).

21. Schäfer, A. et al. Small mobilizable multi-purpose cloning vectors derived from the Escherichia coli plasmids pK18 and pK19: selection of defined deletions in the chromosome of Corynebacterium glutamicum. Gene 145, 69-73 (1994).

22. Johnson, C. W. \& Beckham, G. T. Aromatic catabolic pathway selection for optimal production of pyruvate and lactate from lignin. Metab. Eng. 28, 240-247 (2015).

23. Delaney, N. F. et al. Development of an optimized medium, strain and high-throughput 
culturing methods for Methylobacterium extorquens. PLoS One 8, e62957 (2013).

344 24. Sniegowski, P. D., Gerrish, P. J. \& Lenski, R. E. Evolution of high mutation rates in experimental populations of E. coli. Nature 387, 703-705 (1997).

25. Johnson, C. W. et al. Enhancing muconic acid production from glucose and lignin-derived 